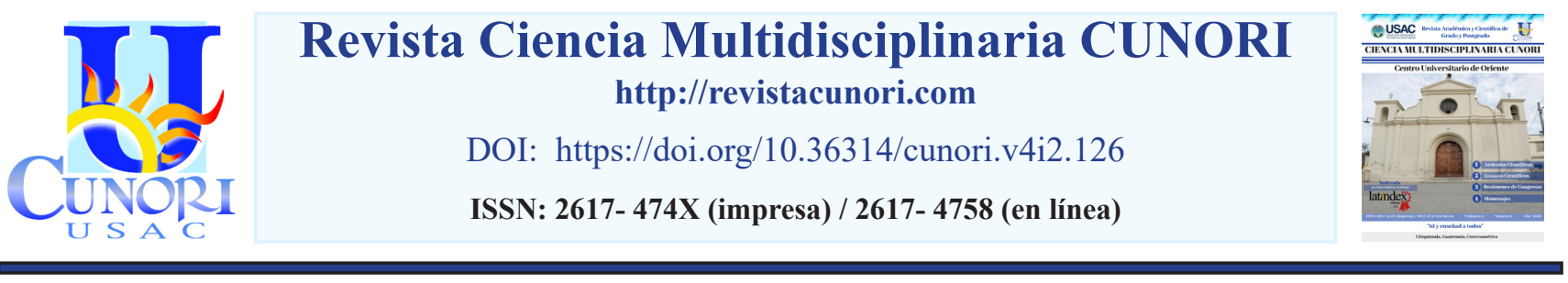

Referencia del artículo

Linares-Díaz, A. J. y Miranda-Vivar, F. M. (2020). Clenbuterol en la carne bovina comercializada en la cabecera departamental de Chiquimula, Guatemala. Revista Ciencia Multidisciplinaria Cunori, 4(2), 39-46. https://doi.org/10.36314/cunori.v4i2.126

\title{
Clenbuterol en la carne bovina comercializada en la cabecera departamental de Chiquimula, Guatemala
}

\section{Clenbuterol in bovine meat sold in the departmental capital of Chiquimula, Guatemala}

\author{
Alejandro José Linares Díaz y Flor María Miranda Vivar \\ Centro Universitario de Oriente de Universidad de San Carlos de Guatemala \\ https://orcid.org/0000-0001-9629-9948 \\ Recibido: 13 de abril de 2020 / Revisado: 17 de abril de 2020 / Aceptado: 25 de mayo de 2020 \\ Disponible en internet el 30 de octubre de 2020 \\ *Autor para correspondencia. \\ Correo electrónico: alinaresdiaz@yahoo.es
}

\section{Resumen}

Q1 presente artículo, parte de la importancia de determinar la presencia de clorhidrato de clenbuterol en la carne bovina, que se - comercializan en la cabecera departamental de Chiquimula, con el fin de determinar si la sustancia es utilizada como estimulante y promotor de crecimiento en ganado, aun cuando existe prohibición nacional, en su uso para cualquier fin, determinando una alta presencia de la sustancia en las muestras analizadas. Adicionalmente, se hizo un estudio de opinión en la población del municipio de Chiquimula, para determinar el nivel de conocimiento sobre la presencia, uso y riesgo a la salud pública, derivados del consumo de carne bovina con clorhidrato de clenbuterol. Se encontró una proporción considerable de casos positivos en las pruebas de laboratorio, situación que demuestra, el uso indiscriminado del componente, en dietas para animales en sistemas de ganado bovino de engorde. El estudio de opinión, demostró además que, existe muy poco conocimiento sobre lo que es el clenbuterol, los usos del mismo en la alimentación animal y los posibles riesgos por el consumo de carnes que posean algún tipo de concentración del estimulante.

Palabras clave: clenbuterol, carne, bovino, hormona, Chiquimula

\begin{abstract}
$\mathrm{T}$ his article starts from the importance of determining the presence of clenbuterol hydrochloride in bovine meat, which are marketed in the departmental capital of Chiquimula, in order to determine if the substance is used as a stimulant and growth promoter in cattle, even when there is a national prohibition, in its use for any purpose, determining a high presence of the substance in the samples analyzed. Additionally, an opinion study was made in the population of the municipality of Chiquimula, to determine the level of knowledge about the presence, use and risk to public health, derived from the consumption of bovine meat with clenbuterol hydrochloride. A considerable proportion of positive cases were found in laboratory tests, a situation that demonstrates the indiscriminate use of the component in animal diets in cattle fattening systems. The opinion study also showed that there is very little knowledge about what is the clenbuterol, its uses in animal feed and the possible risks of consuming meats that have some type of stimulant concentration.
\end{abstract}

Keywords: clenbuterol, meat, bovine, hormone, Chiquimula 


\section{Introducción}

Guatemala es un país con extensión territorial apta y suficiente, para la crianza y engorde de ganado de diversos tipos. El sector productivo bovino, ha mostrado cambios importantes a nivel de mercado, donde se demanda una gran cantidad de carne y en aumento, principalmente a nivel urbano dado que, en el contexto rural, la situación de pobreza e inseguridad alimentaria y nutricional, no permite incluir este tipo de alimentos en la dieta. De acuerdo al Ministerio de Economía -MINECO- (2019), la producción de carne de res ha tenido fluctuaciones entre 70 y 75 toneladas métricas por año, del 2014 al 2019, mientras el consumo doméstico tuvo una tendencia de incremento positiva, aumentando de 81 a 98 toneladas métricas anuales, para los mismos años.

Lo anterior muestra que, si bien, la oferta ha sido variable en los últimos 5 años, probablemente por influencia del cambio climático, el consumo doméstico si se incrementó en un $22 \%$. De hecho, según Carlos Zúñiga, presidente del comité permanente de la Semana del Ganadero y Expoleche 2014, afirma que, Guatemala tiene un consumo per cápita de cuatro kilos de carne de res, un poco menos de la cuarta parte del consumo reportado en Costa Rica, que es de 17 kilos al año por persona (Larios, 2014). Por esta razón, uno de los principales retos es encontrar mecanismos para satisfacer este incremento en la demanda de carne bovina, más aún, cuando la Política Agropecuaria 2016-2020 del Ministerio de Agricultura, Ganadería y Alimentación -MAGA-, fija sus acciones en fortalecer las actividades agropecuarias que satisfagan las necesidades de alimentación en la población guatemalteca, para el cumplimiento de las responsabilidades sectoriales basados en los ejes: seguridad alimentaria y nutricional, salud pública, desarrollo económico productivo y desarrollo social.

El cumplimiento de este objetivo, ha llevado a algunos productores a encontrar ventajas competitivas que, en ocasiones escapan a la normativa vigente, como el Acuerdo Ministerial 821-2007 del MAGA (2007) que prohíbe el registro, uso y comercialización del clenbuterol en todas sus presentaciones, especialmente en el ramo pecuario dado que, de forma clandestina se ha usado como promotor de ganancia de peso en el engorde de ganado bovino, por su importante efecto anabolizante que, permita obtener una mayor cantidad de carne (hasta un 40\% más de masa muscular, según Valladares, 2015), con menor contenido de grasa (hasta un 8\% menos de grasa, según Flores, 2010), en el menor tiempo y costo posible.

De hecho, la propiedad de aumento de la masa muscular en los animales destinados al sacrificio, ha hecho del clorhidrato de clenbuterol, uno de los compuestos más popularmente utilizados por los productores ganaderos sin responsabilidad social, debido a que es una sustancia que presenta una serie de efectos tóxicos si se consume de forma descontrolada y de dosis mínimas a elevadas (FAO; OMS, 2017). Esto genera diversos problemas: falta de garantía en la calidad de los insumos para el productor, diferencias en los modelos de administración de fincas ganaderas e incapacidad competitiva para quienes no administren sustancias anabolizantes, informalidad en el sector productivo ganadero por ausencia de rastreabilidad y fiscalización, además de, falta de información al consumidor en cuanto a la calidad, las características, las condiciones y los riesgos, derivados del consumo de carne bovina y subproductos de la misma. 
Es poca la información existente a nivel nacional, en cuanto al uso del clorhidrato de clenbuterol para el engorde de ganado bovino y, aunque legalmente, su uso está prohibido, la administración indiscriminada de la sustancia, conlleva a problemas a la salud del animal y del consumidor de la carne, apoyados en la teoría fundamentada, que le considera como una sustancia con potencial cancerígeno y toxicológico. En la región oriental de Guatemala, especialmente en el departamento de Chiquimula, la actividad ganadera bovina se constituye en uno de los principales pilares productivos a nivel agropecuario, dado las condiciones climáticas, las actividades familiares y que, geográficamente es un punto trifronterizo para la comercialización de animales en pie y en canal, provenientes de diferentes puntos de Guatemala, El Salvador y Honduras.

Por lo tanto, existe diversidad en el manejo productivo y nutricional de los hatos del departamento, lo que supone que, en una proporción de los mismos, se ha utilizado el clorhidrato de clenbuterol en el engorde de ganado, pero, no había información al respecto por la prohibición legal en cuanto a su uso. Tomando como referencia que, para el año 2018, la comercialización de productos cárnicos en Guatemala se realizó a través de tiendas pequeñas independientes (carnicerías) en un $74.2 \%$, seguida por los supermercados en un $9.3 \%$, tiendas de descuentos en un $9.2 \%$, tiendas especializadas de comida en un $3.4 \%$, hipermercados en un $1.9 \%$ y tiendas de conveniencia en un $1.9 \%$ (MINECO, 2019).

Este trabajo, analizó 58 muestras de carne bovina comercializada en los expendios de los mercados municipales de la cabecera departamental de Chiquimula, así como expendios externos registrados. Por medio del análisis de cromatografía líquida de alta resolución acoplada a espectrometría de masas (HPLC-MSMS), se encontró presencia de clorhidrato de clenbuterol un total de $51.72 \%$ de las muestras. Dicho valor refleja el elevado uso del anabolizante en el engorde del ganado comercializado en la zona, con los respectivos conflictos relacionados a la salud pública. Además de determinar el uso del anabolizante, el estudio determinó el nivel de conocimiento de la población consumidora, del uso del anabolizante y sus posibles riesgos a la salud derivados del consumo de carne, mostrando que solo el $42.64 \%$ de la población encuestada, tiene algún grado de conocimiento de qué es el clorhidrato de clenbuterol, un $5.88 \%$ saben de su uso en la alimentación de ganado bovino y apenas, un $2.95 \%$ conoce sobre el riesgo a la salud pública humana. De esta manera, la producción de carne bovina en la cabecera departamental de Chiquimula, en ausencia a la inspección de la normativa vigente, hace que el clorhidrato de clenbuterol residual en carne, llegue al mercado final que, en su mayoría sin saberlo, al consumirla pueden dar paso a intoxicaciones importantes que, pueden afectar directamente su salud de forma imprevisible.

\section{Materiales y métodos}

El estudio lo realizó la estudiante Flor María Miranda Vivar con la colaboración del Maestro Alejandro José Linares Díaz y la asesoría del maestro Carlos Suchini Ramírez en la cabecera departamental de Chiquimula, en Guatemala. La misma está ubicada en el oriente del país, a 170 kilómetros de la capital guatemalteca, limitando al norte con el departamento de Zacapa, al sur con la República de El Salvador y el departamento de Jutiapa, al este con la República de Honduras y al oeste con los departamentos de Jalapa y Zacapa. (MINECO, 2019). 
Se realizó una investigación exploratoria con enfoque cualitativo, bajo un proceso sistemático y ordenado, distribuido en dos procesos: 1) el análisis de las muestras cárnicas bovinas de 25 expendios de carne en los mercados municipales y 3 expendios externos, con un doble muestreo con 30 días de separación (meses de agosto y septiembre del 2019); y 2) un estudio de opinión en 68 familias (determinadas según muestreo aleatorio para poblaciones finitas), sobre el conocimiento en la población de la cabecera departamental de Chiquimula, sobre el uso y los efectos del consumo de carne bovina con clorhidrato de clenbuterol.

En análisis de las muestras cárnicas, fue realizado a través de la técnica de cromatografía líquida de alta resolución acoplada a espectrometría de masas (HPLC-MSMS), ejecutado por el Laboratorio Fitosanitario del Ministerio de Agricultura, Ganadería y Alimentación -MAGA-, ubicado en Bárcenas, municipio de Villa Nueva, departamento de Guatemala, determinando la presencia o ausencia de clorhidrato de clenbuterol en la muestra. El estudio de opinión, se realizó a través de una encuesta, que permitió recopilar características sociodemográficas, de consumo de carne bovina y el nivel de conocimiento del uso y riesgos del anabolizante, analizando los datos a través de estadísticas descriptivas multivariadas.

\section{Resultados}

\section{a. Determinación de clorhidrato de clenbuterol en muestras de carne bovina}

Los resultados obtenidos sobre la presencia y concentración de clorhidrato de clenbuterol, en la carne que se expende para consumo en la cabecera departamental de Chiquimula, se muestra en la siguiente tabla:

Tabla 1. Presencia y concentración de clorhidrato de clenbuterol en muestras cárnicas bovinas

\begin{tabular}{cccccccccc}
\hline \multirow{2}{*}{ Lugar } & \multicolumn{3}{c}{ Primer muestreo } & \multicolumn{3}{c}{ Segundo muestreo } \\
\cline { 2 - 9 } & $\begin{array}{c}\text { No. } \\
\text { muestras }\end{array}$ & Positivas & $\begin{array}{c}\text { Porcentaje } \\
(\%)\end{array}$ & $\begin{array}{c}\bar{x} \\
(\mathbf{p p b})\end{array}$ & $\begin{array}{c}\text { No. } \\
\text { muestras }\end{array}$ & Positivas & $\begin{array}{c}\text { Porcentaje } \\
(\%)\end{array}$ & $\begin{array}{c}\bar{x} \\
(\mathbf{p p b})\end{array}$ \\
\hline $\begin{array}{c}\text { Mercado } \\
\text { Central }\end{array}$ & 15 & 10 & 63 & 0.64 & 15 & 11 & 69 & 0.63 \\
$\begin{array}{c}\text { Mercado } \\
\begin{array}{c}\text { Terminal } \\
\text { Comercios } \\
\text { externos }\end{array}\end{array}$ & 03 & 06 & 60 & 0.52 & 10 & 03 & 30 & 0.60 \\
\hline
\end{tabular}

Fuente: Miranda-Vivar (2020). Con información del estudio de campo.

En los expendios de carne del mercado central se reportaron 10 casos positivos (63\% de las muestras), en el mercado de la terminal se encontraron 6 casos positivos $(60 \%)$; sin embargo, en los comercios externos a los mercados municipales, las carnes que se expenden no reportan presencia de clorhidrato 
de clenbuterol. En el segundo muestreo realizado a los mismos comercios, los resultados obtenidos reflejan la presencia de anabólico, en 11 expendios del mercado central (69\%), 3 en mercado de la terminal (30\%) y ningún caso en los comercios externos.

\section{b. Estudio de conocimiento y opinión del clorhidrato de clenbuterol}

En la investigación realizada, se encuestaron a un total de 68 personas representantes de familias, conformadas por núcleos de 4-7 miembros en el 67.64\%, seguido de 1-3 miembros en el 23.52\% y más de 7 miembros en el $8.82 \%$ de los casos. En cuanto al hábito de consumo de carne de res, el 85.28\% de los encuestados, afirmaron que si la consumen mientras que un $14.70 \%$ respondió negativamente a la pregunta. En cuanto a la frecuencia de consumo, el $72.41 \%$ reportaron consumir de forma semanal, un $12.06 \%$ de forma quincenal y un $15.51 \%$ de forma mensual u ocasional.

El factor más importante, con respecto a los hábitos de consumo es la cantidad de producto consumido, donde un $72.41 \%$ reportaron consumir de 1 a 3 libras de carne $(0.45-1.36 \mathrm{~kg})$ y un $27.58 \%$ consumen más de 4 libras $(1.81 \mathrm{~kg})$, en períodos que van de 1 semana a ocasionalmente, valores que coinciden a los presentados a nivel nacional (4-7 kg/habitante/año), tomando en cuenta el número de miembros por núcleo familiar y la frecuencia de consumo reportada. En lo que se refiere al conocimiento del clorhidrato de clenbuterol, el $42.64 \%$ de las personas encuestadas indicaron conocer sobre la existencia y uso de la sustancia anabolizante mientras que, el $57.35 \%$ no tienen conocimiento sobre el tema. De igual manera, al preguntárseles si conocían sobre los usos del anabólico, solamente un 14.70\% afirmó conocer el uso del mismo en la producción animal, mientras que un $85.29 \%$ respondieron de forma negativa al cuestionamiento.

\section{Discusión}

\section{a. Determinación de clorhidrato de clenbuterol en muestras de carne bovina}

De las muestras carnícas, el 51.72\% de las carnes bovinas comercializadas en la cabecera departamental de Chiquimula, fueron positivas a la prueba HPLC-MSMS para clorhidrato de clenbuterol, en concentraciones promedio de 0.596 partes por billón (ppb). En ese contexto, la cantidad de muestras cárnicas positivas a la presencia del clorhidrato de clenbuterol fueron en promedio general de $51.72 \%$, parámetro que está por encima de lo encontrado por Ortíz Borges, et al. (2005), que reportó en el Estado de Mérida, México mediante la misma técnica de laboratorio, presencia en el $37.5 \%$ en 24 muestras tomadas directamente en rastros. Sin embargo, los valores encontrados del anabolizante fueron de 2.76 ppm (2760 ppb), valores muy por encima del encontrado en este estudio que fue de 0.596 partes por billón (ppb), sin embargo, esto puede deberse a que los niveles máximos se alcanzan de 18 a 65 horas posterior a la administración del clorhidrato de clenbuterol al animal, por lo que las muestras tomadas del rastro en el estudio de Ortíz Borges, et al., presentaron por lógica mucho mayor presencia que las del presente estudio. 
De igual manera Olivares, et al. (2015), estudio la presencia de clenbuterol en tejido muscular bovino en rastros en la región de Tierra Caliente, Estado de Guerrero, México, pero a través de una técnica denominada: Rapid test, que solo identifica positivos o negativos a la presencia del anabolizante, pero no determina la cantidad presente en la muestra. Dicho estudio, reportó en un 38.24\% de 34 muestras que sí existía Clorhidrato de Clenbuterol. Dato menor al encontrado en este estudio, cuya técnica se cataloga como confirmatoria a métodos de cuantificación como ELISA. A nivel nacional, Chávez Maldonado (2017), en su estudio realizado en mercados municipales de 4 localidades del departamento de Suchitepéquez determinó que, las muestras fueron positivas a la presencia de clorhidrato de clenbuterol en un $42.5 \%$ de las muestras con concentraciones mayores promedio de $2.0733 \mu \mathrm{g} / \mathrm{kg}$ o ppb, representando un $347.86 \%$ más de las concentraciones encontradas en el presente estudio, lo que pudiera indicar que la cantidad de clorhidrato de clenbuterol suministrada en la dieta a los animales de la región oriental (incluído el departamento de Chiquimula), es menor a la brindada en zonas sur-occidentales del país.

\section{b. Estudio de conocimiento y opinión del clorhidrato de clenbuterol}

El consumo de carne bovina en el $85.28 \%$ de la población encuestada, en su mayoría de forma semanal y en cantidades importantes, permite afirmar que, existe un uso considerable de carne de res en la cabecera departamental de Chiquimula (hasta $12.25 \mathrm{~kg} /$ persona/año), valor similar al promedio de consumo mundial de $11.67 \mathrm{Kg} /$ persona/año y un poco menor al obtenido en un estudio de la demanda y consumo de carne bovina en la cabecera municipal de Chiquimula, donde informa de un consumo promedio de $15.65 \mathrm{Kg} /$ persona/año (Villafuerte, 2011). Si bien, un poco menos de la mitad de la población reportaron conocer la existencia del clorhidrato de clenbuterol, solo una sexta parte reportó saber del uso en el engorde. Esto demuestra no solo que no existe un conocimiento suficiente en la población, sobre lo que es el clorhidrato de clenbuterol, sino que aún entre los que afirmaron conocer lo que es, manifiestan desconocer sus usos.

En otros términos, las personas en la zona consumen la carne bovina, sin conocer los riesgos potenciales a los que se expone y las repercusiones en su salud, puesto que solamente un $5.88 \%$ de los encuestados reportaron conocer los efectos del clorhidrato de clenbuterol en el animal y solo un $2.95 \%$ en los efectos y riesgos a la salud del consumidor. Esto afirma aún más, la preocupante situación de desconocimiento general de la población estudiada sobre del uso y efecto del anabólico en la alimentación del ganado bovino y la posible presencia en las canales consumidas, sin embargo, al consultarles que si tuvieran certeza de cuáles carnes poseen residuos del anabólico y cuáles no, el 100\% de los encuestados aseveraron que no consumirían las carnes positivas.

\section{c. Recomendaciones finales}

Por lo anteriormente expuesto, se recomienda socializar la información de las diferentes investigaciones relacionadas al tema del uso indiscriminado de anabólicos y promotores de crecimiento en la producción animal, ante las instancias gubernamentales como el Ministerio de Agricultura, Ganadería y Alimentación, con la finalidad de generar planes de vigilancia y monitoreo del uso de sustancias prohibidas en las explotaciones pecuarias. 
También, concientizar a la población guatemalteca y a todos los involucrados en la cadena productiva, de los riesgos y efectos del uso de sustancias como el clorhidrato de clenbuterol en la producción animal, e impacto a la salud pública. Por último, considerar para próximos estudios de determinación de presencia de clorhidrato de clenbuterol u otro anabolizante en piezas cárnicas, el lugar de procedencia de los animales sacrificados, con el fin de identificar el sistema y ubicación predominante en este tipo de prácticas.

\section{Agradecimientos}

Al asesor Carlos Suchini Ramírez, a la M.V. Khrista Polanco, por su apoyo técnico e institucional a través del Ministerio de Agricultura, Ganadería y Alimentación, en la ejecución de esta investigación. Al M.V. Eliot Montenegro, por su apoyo técnico e institucional a través del Laboratorio de Diagnóstico Fitosanitario del Ministerio de Agricultura, Ganadería y Alimentación, para realizar las pruebas por el método HLPC-MSMS. Al Ministerio de Agricultura, Ganadería y Alimentación -MAGA-, por su apoyo técnico y financiero en la realización de 58 pruebas para determinación de clorhidrato de clenbuterol en muestras cárnicas bovinas.

\section{Referencias}

Chávez Maldonado, Ana L. 2017. Detección de niveles de clenbuterol en carne bovina comercializada en mercados municipales de Suchitepéquez. Tesis de grado. Suchitepéquez, Guatemala, CUNSUR. 105 p. http://www.repositorio.usac.edu.gt/7892/1/TESIS\%20.pdf

Flores Ayala, Daniel. 2010. Uso del clenbuterol en bovinos de carne y su importancia en salud pública. Tesis de grado. Torreón, Coahuila, México, UAAAN. 70 p. http://repositorio.uaaan. mx:8080/mlui/bitstream/handle/123456789/7945/DANIEL\%20FLORES\%20AYALA. pdf? sequence $=1 \&$ isAllowed $=\mathrm{y}$.

Larios, Roxana. 2014. Consumo de carne y leche es bajo en Guatemala. Prensa Libre, Guatemala; 11 junio 2014. https:/www.prensalibre.com/economia/carne-consumo-semana_del_ ganadero_y_expoleche_0_1154284805-html/

MAGA (Ministerio de Agricultura, Ganadería y Alimentación). 2017. Acuerdo ministerial no. 821-2007. Guatemala. 2 p. https://sistemas.maga.gob.gt/normativas/ Normativas?page $=2 \&$ sortOrder $=$ Resumen_desc \&categoriaId $=4$

Ministerio de Economía -MINECO-. 2019. Área de Inteligencia de Mercado, Guatemala. Industria de cárnicos en Guatemala. 25 p. https:/www.mineco.gob.gt/sites/default/files/carnicos_en_guatemala_13_1.pdf

Olivares, J.; Quiroz, F.; Rojas, S.; Camacho, L.M.; Cipriano, M. 2015. Determinación de clembuterol en tejido muscular en bovinos en rastros de la región de Tierra Caliente del estado de Guerrero. Revista de Energía Química y Física 2(4):338-342. http://www.ecorfan.org/bolivia/researchjournals/ Energia_Quimica_y_Fisica/vol2num4/Revista_de_Energia_Quimica_y_Fisica-v2_N4-8-12.pdf

Organización de las Naciones Unidas para la Agricultura y la Alimentación -FAO-. 2017. Máximos de residius (LMR) y recomendaciones sobre la gestión de riesgos (RGR) para residuos de medicamentos veterinarios en los alimentos CAC/ MRL 2-2018. Roma, Italia, FAO/ OMS, Codex 
Alimentarius. 42 p. http://www.fao.org/fao-who-codexalimentarius/sh-proxy/ar/?lnk=1\&url=https $\% 253 \mathrm{~A} \% 252 \mathrm{~F} \% \quad$ 252Fworkspace.fao.org $\% 252 \mathrm{Fsites} \% 252 \mathrm{Fcodex} \% 252 \mathrm{FStandards} \% 252 \mathrm{FCA}$ C\%2BMRL\%2B2\%252FMRL2s.pdf

Valladares-Carranza, B.; Bañuelos-Valenzuela, R.; Peña-Betancourt, S.D.; Velásquez-Ordonez, V.; Echavarría-Chaires, F.G.; Muro-Reyes, A.; Zaragoza-Bastida, A.; Ortega-Santana, C.; Zamora-Espinosa, J.L.; Gutiérrez Castillo, A. 2015. Implicaciones del uso de clorhidrato de clenbuterol en la producción pecuarias. Revista Electrónica de Veterinaria 16 (2): 4-6. https://www.redalyc.org/ pdf/636/63641398007.pdf

Villafuerte Villeda, Hugo. 2011. Estudio de mercado de la carne de ganado bovino en la cabecera municipal de Chiquimula, departamento de Chiquimula, Guatemala. Tesis de Maestría. Chiquimula, Guatemala, Usac-Cunori. 121 p. http:/www.repositorio.usac.edu.gt/ 3412/1/19\%20MAEA\%20 TG-1739-1120.pdf

\section{Sobre el Autor}

\section{Alejandro José Linares Díaz}

es Zootecnista en el grado académico de Licenciatura y Maestro en Educación con Orientación en Medio Ambiente egresado del Centro Universitario de Oriente de la Universidad de San Carlos, actualmente está en proceso de graduación en el Doctorado en Investigación para el Desarrollo Social en USAC/CUNSURORI. Se ha desempeñado como asesor de trabajos de graduación en grado de Licenciatura en temas de Pastos y Forrajes, Microbiología animal y de la carne, Inocuidad de los alimentos y Cunicultura. Ha realizado diversas investigaciones en proyectos de CRIA/IICA Región Oriente, cadena bovinos de doble propósito en CATIE-IICA-USDA. Es delegado representante del Centro Universitario de Oriente ante el programa de Investigación en Alimentación y Nutrición de la Dirección General de Investigación - DIGI/USAC. Es profesor y Asesor del curso de Investigación en la Maestría en Formulación y Evaluación de Proyectos con énfasis en Impacto Ambiental USAC/CUNZAC. Tiene artículos científicos publicados en la Revista del Sistema de Estudios de Postgrado -SEP- de la USAC, Revista Académica Cunzac, Revista Ciencia Multidisciplinaria Cunori y en la Revista Perspectivas Rurales de la Universidad Nacional de Costa Rica.

Copyright (c) Alejandro José Linares Díaz, Flor María Miranda Vivar

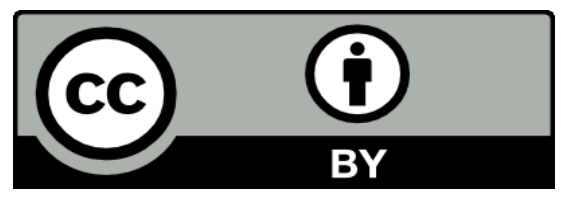

Este texto está protegido por una licencia CreativeCommons 4.0.

Usted es libre para compartir, copiar y redistribuir el material en cualquier medio o formato y adaptar el documento, remezclar, transformar y crear a partir del material para cualquier propósito, incluso comercialmente, siempre que cumpla la condición de atribución: usted debe reconocer el crédito de una obra de manera adecuada, proporcionar un enlace a la licencia, e indicar si se han realizado cambios. Puede hacerlo en cualquier forma razonable, pero no de forma tal que sugiera que tiene el apoyo del licenciante o lo recibe por el uso que hace. 